\title{
Systems Engineering in Terms of Exergy
}

\author{
José A. Camberos and David J. Moorhouse \\ Multidisciplinary Technologies Center, Air Vehicles Directorate U. S. Air Force Research Laboratory, \\ Wright-Patterson AFB, OH 45433, USA
}

Correspondence should be addressed to José A. Camberos, jose.cambero@wpafb.af.mil

Received 11 March 2009; Accepted 21 July 2009

Recommended by Hui Hu

We address the design of a flight vehicle from the viewpoint of a system of systems and we discuss the integration of the individual technical disciplines. Then a conceptual fundamental methodology and tools required for the analysis, design, and optimization of aerospace vehicles in terms of the efficient use of on-board energy are discussed. This suggests changing the design paradigm to the optimization of a system of energy systems. We propose a foundation for system-level design with optimization based on minimum exergy destruction.

Copyright ( $) 2009$ J. A. Camberos and D. J. Moorhouse. This is an open access article distributed under the Creative Commons Attribution License, which permits unrestricted use, distribution, and reproduction in any medium, provided the original work is properly cited.

\section{Introduction}

Aircrafts have evolved to a point where they are extremely complex machines posing a highly integrated design problem. A military vehicle includes many systems that are all interrelated and dependent on power (or energy) in some form. In some of the systems there is also the creation of by-products, in the form of heat energy, that have to be removed from that equipment. There obviously exist methods for the design of all these systems, based on the evolutionary nature of vehicle development. However, the more we depart from existing data bases and experience levels, the less confidence we can have that we are close to an optimal design. In addition, many of the classical techniques are based on simplifying assumptions that were used in the original derivation. If these are not considered, then there is no guide to when those classical techniques no longer give an acceptable solution.

The need exists for a methodology that can support design of the complete vehicle as a system of systems in a common framework. It must allow consideration of all aspects in terms of common metrics in order to conduct fully credible trades. The vision is to develop such a methodology that will support all required levels of design activity in a natural fashion, from conceptual comparisons through the final configuration and lead to a true system-level optimized design. In nature, if something is inefficient, then it dies out or it adapts to the environment. We claim that this "inefficiency" can be quantified in terms of useful work versus energy wasted. We also consider an aerospace system as consuming fuel and doing work in some form. Lower specific fuel consumption of the engine is one dominant factor, but when this is considered completely separated from vehicle application, then the system will probably not be truly optimized. Therefore, there is a need for tools and processes enabling the discovery of new and innovative configurations by designing for maximum efficiency and minimum energy waste at the overall optimum system design level, subject to the appropriate constraints of course [1].

We suggest that the first question in the design process of any flight vehicle should be whether the solution is expected (or desired) to be evolutionary or revolutionary. Evolutionary can be defined as applying to concepts for which there is significant existing data on "similar" configurations. There is then some confidence in the extrapolation of that data. A revolutionary concept, with no prior flight experience, requires a more rigorous analytical process. Any application within these extremes will differ in detail, but the object of this paper is to suggest that a system-level process for integrating the various technical disciplines requires a "common currency", which is exergy.

\section{Background}

Consider the process for a new aircraft design. The concurrent design process relies on some form of system analysis or 
systems engineering [1]. The "Systems Engineering Process" has been expressed as follows:

(1) break the system down into component parts,

(2) understand each individual part,

(3) determine how the parts interact,

(4) define the contribution of each to system performance,

(5) put the system back together,

(6) build it when the analysis shows that the design meets requirements.

Written in this form, then Systems Engineering seems like a straightforward sequential process. So we can ask what are the problems with Systems Engineering?

(1) Breaking the system down into component parts has been done very well historically. It has produced well-defined disciplines in scientific areas such as propulsion, aerodynamics, structural design, flight control, and subsystems. It is commonly accepted that many of the analytical methods are based on the laws of physics, even with simplifying assumption and empirical adjustments. When we consider "the laws of physics", however, it must be recognized that those laws are frequently written in a form suitable for a specific application. There may be assumptions in content and formulation which could affect other applications. A trivial example can be used to start this thinking process.

For an aircraft in cruise, lift must be equal to weight. So that, if $L-W=0$, then there is zero work being done in the vertical axis. Correct from a certain viewpoint, however: consider the same problem from a different viewpoint. Draw a control volume around that cruising vehicle. The "system" \{undefined as yet\} inside that control volume is doing work to create a vertical force to counter the external force of gravity. So, how is that work being done?

In one theory, that vertical force is created by giving vertical momentum to a quantity of freestream air as the vehicle passes through it. The "laws of physics", however, are written in the format of air flowing horizontally into the control volume and leaving with some vertical velocity. Force equals rate of change of momentum, exact physics.

The above discussion helps little in designing a flight vehicle: it is the engine "that is doing all the work" in cruise. So, the practical physics is to analyze the vertical work in terms of induced drag. This was done with assumptions to get an analytical solution followed by adjustments to match "real data". If we simplify conventional aircraft design, the process was to optimize the various parts, then build multiple prototypes against a set of requirements and fly them to choose the winner.

(2) Understanding each individual part might have been done too well! Individual technical disciplines are quite well understood, although obviously not in a $100 \%$ accurate sense. Research is still ongoing in all disciplines. At the risk of over-simplifying the discussion, the old fashioned methods developed a good process for steps (1) and (2). It was not uncommon to minimize the efforts on steps (3) and (4) and then move on to step (5) and build a prototype and fly it to see if it met requirements.

(3) Determining how the parts interact is not a straight forward process, and interactions are often found in flight test; for example, see Norton. One example of progressing past understanding the "individual part" for aircraft design is the Multidisciplinary Optimization (MDO) aspect. It started as efforts to integrate the calculation of aerodynamic loads into the structural optimization, but this is still calculation of the forces acting on the structure. It was not really a change in the structural design paradigm, but this field is also expanding, for example, by inclusion of control aspects. We thus have a question of how to define the interactions and how to assess the significant interactions versus the negligible. The appropriate answer can be expected to be unique for different applications.

(4) Defining the contribution of each component to system performance is a continually improving process while the systems are becoming exponentially more complex, as are the interactions. But we pose the question of what system metric is appropriate if components are designed (optimized) to different aspects. Also, what system model is needed for any particular aspect? What is the necessary level of fidelity?

(5) Putting the system back together in the ideal sense would require a complete physics-based model. In the practical world, however, there are many questions to be addressed and many potential problems. How have the components been validated as being ready for system integration? One part of the preceding question is what data is experimental and what is analytical. It should not be assumed that any of the data is completely accurate, so that an explicit accounting of uncertainties is required. How have the component disciplines been coordinated during the design process? Linear superposition may be sufficiently accurate in some aspects and totally wrong for others.

(6) The final aspect, that is, build it when the analysis shows that the design meets requirements, raises many questions. What is the appropriate analysis or should it be analyses? The answer to this question may be different for any application. There may be the exception for minor evolutionary upgrades to an existing system, but even that should not be taken for granted. This question leads into the following section.

As a final comment for this section, we strongly suggest that "System Engineering" should not be applied as a sequential process, but as an integrated and continual iterative process.

\section{System Design}

Great many physical problems have been solved at this stage of flight vehicle evolution. As previously stated, "the laws of physics" are frequently written in a form suitable for a specific application, and that application has not been the complete system. Considering a flight vehicle as device to do work (expanding the approach by the first author, Moorhouse [2]) we can address the issue of energy efficiency. The term in the title "Exergy" can be defined as the available work from an 
energy source. With fuel as the energy source to accomplish all the required work, then "everything on the vehicle" must be defined in those terms. The weight of the fuel itself is part of the overhead, and the concept of a multiplication factor at the vehicle level for fuel saved is well known. The reverse is that any change in heat content of a fuel must be balanced against possible changes in fuel weight or volume, that is, aircraft size and weight. This becomes even more of a design consideration if we are using unconventional fuels or mixed cycle operation In addition, the fuel may be used as a cooling medium and, therefore, a critical part of the aircraft thermal energy distribution and management system. The authors are part of a program for developing such methods for the design of a flight vehicle as a complete system of energy systems. Next we review some contributions that have been made as part of that AFRL program.

3.1. Design Optimization. As we have discussed, consideration of a system does require the design team to address the components. A critical part of this was the development of a decomposition strategy where all the subsystem components can be optimized to a system-level metric; see Munoz and von Spakovsky [3] and Rancruel and von Spakovsky [4-8].

Periannan et al. [9] used the above technique to show the advantages of applying exergy-based analysis and optimization methods to the synthesis/design and operation of aircraft systems. They compared different objective functions for the same design, minimizing takeoff gross weight, maximizing thrust efficiency, maximization of thermodynamic effectiveness, and minimizing exergy destruction. They showed that, as long as the constraint space is the same, an energy-based objective produces the same optimum as that of the exergy-based objective provided that they are equivalent forms of the same thing, for example, fuel consumption. With the active (i.e., with losses and degrees of freedom) inclusion of the AirFrame SubsystemAerodynamics (AFS-A), which is by definition not an energy system in the traditional sense, this equivalency between energy and exergy objectives properly interpreted no longer appears to hold. It is in fact the lack of a common currency, which is the case when an exergy-based approach is not used, that results in an inability to relate very different types of inefficiencies in one part of a system (such as those in the AFS-A) to those occurring in another part (such as those in the Propulsion System and Environmental Control System) which points generally to the need for exergy as the basis for both analysis and optimization even though for some systems and some problems energy may be sufficient, as shown in Figures 1 and 2.

3.2. High-Speed Considerations. Riggins et al. [10] provide analytical and numerical applications and studies relevant to the vehicle performance-entropy relationship. A simplified configuration of a two-dimensional blunt body at high Mach number was examined utilizing a Navier-Stokes CFD code for cases with forward-facing injection and forwardfacing injection with upstream energy deposition (as heat); these were compared with the base-line case. The entropy

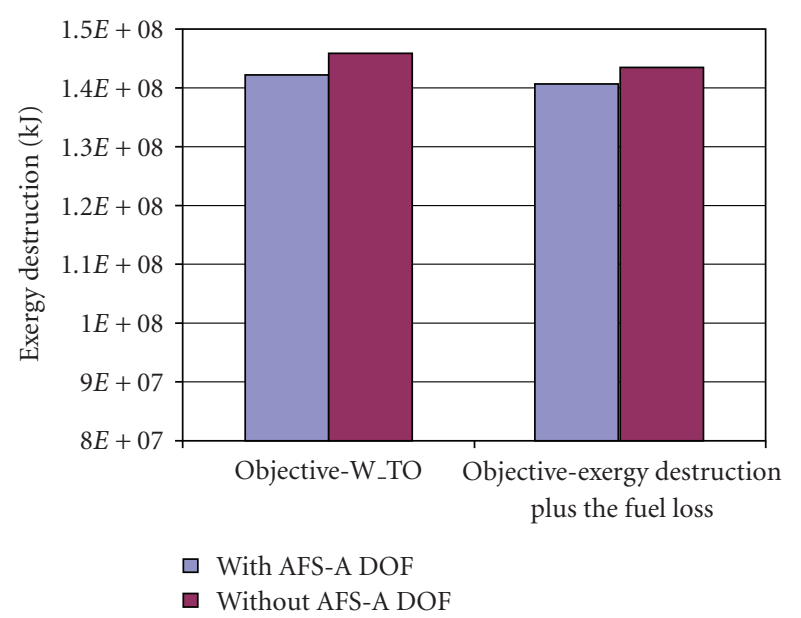

FIGURE 1: Effect of optimization metric on generic high-speed fighter as exergy destruction. Minimizing exergy destruction directly gives the same overall performance as minimizing take-off weight; the advantage with exergy destruction is to identify areas for improvement.

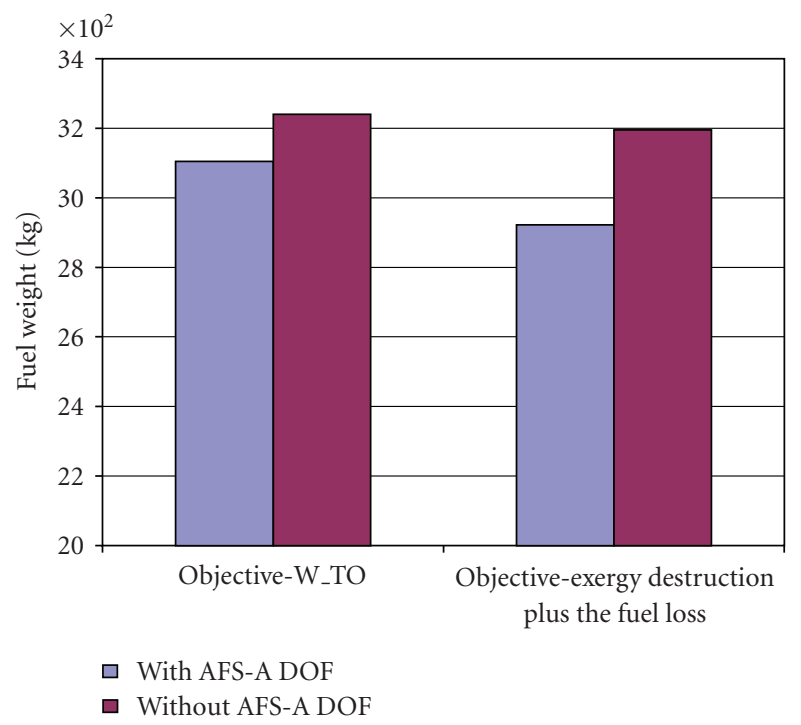

FIGURE 2: Effect of optimization metric on a generic high-speed fighter as fuel weight. Minimizing exergy destruction directly yields a design with improved fuel consumption (less fuel weight).

characteristics of the flow-fields for these cases clarified the relative performance of these cases and demonstrated the utility of the direct link between entropy generation and vehicle performance for configuration analysis, design, and optimization. That concept was then coupled with a simplified rocket-based propulsion system in order to clearly demonstrate the direct relationship between propellant flow rate minimization and minimum overall entropy generation. A second part of the investigation demonstrates the potential advantage of distributing energy in both external airstreams upstream and adjacent to an actual hypersonic vehicle configuration as well as in the "traditional" location of the engine combustor. Figure 3 shows that a strategic use of 


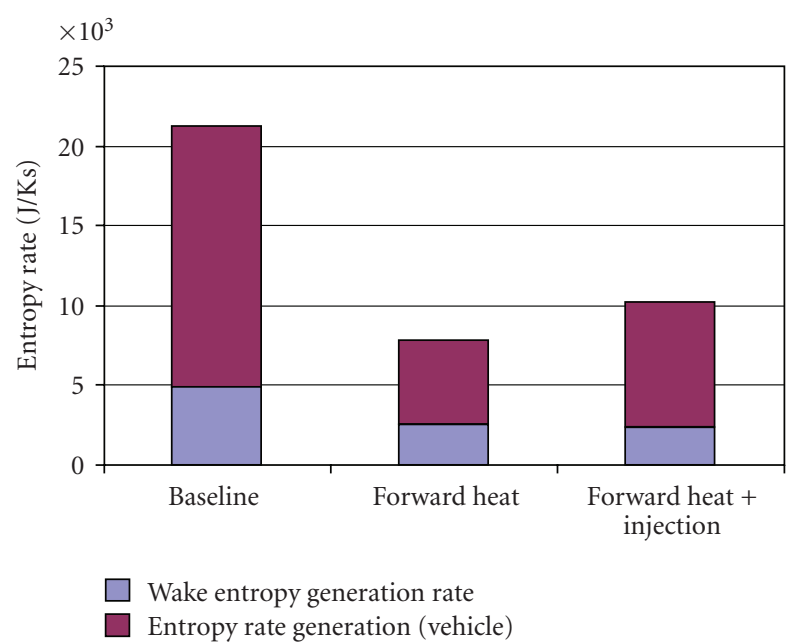

Figure 3: Entropy generation rate: blunt body at flight Mach of 10. The contribution of wake entropy production is significant. However, with strategic use of energy deposition to weaken the shock, total entropy production, and subsequent drag, is reduced.

energy deposition can weaken the stock, thereby reducing total entropy production, and subsequent drag. In this part of the investigation, a second-law analysis was performed on a number of entire vehicle flow-fields and the performance related to the generation of entropy due to flow irreversible losses in both the vehicle flow-field as well as in the vehicle wake. As the authors stated, the major purpose of the overall work was to develop, understand, and apply entropy production as criteria (ultimately "the" criteria) in order to evaluate vehicle performance and performance losses consistently. This forms the basis for the use of a "common currency" in the current vehicle design process- the use of energy concepts examined here is one example of where entropic analysis may be of great use. Such capability may allow the development of new and innovative concepts that do not "just" marginally improve performance but may enable the realization of entire new regimes of performance and operability for high-speed aerospace vehicles.

3.3. Higher Fidelity Computation. Alabi et al. [11, 12] analyzed the entropy generation around a B747-200 aircraft using computational fluid dynamics. The contributions of the various terms in the entropy equation were assessed. Viscous and inviscid models were investigated. The calculations show that inviscid results underestimate the total entropy production by a factor of about 1000 and only capture the entropy generation due to shock and at the stagnation and wake regions. A major difference between the two models was found to be due to the turbulent eddy viscosity present in the viscous calculations. Comparison of the entropy generation terms associated with the viscous calculations shows that the viscous dissipation contributes approximately $90 \%$ of the total entropy production term, with the heat transferrelated term contributing the remaining $10 \%$. In addition, the calculations show possibility of design improvement particularly in the wing sections removed away from the fuselage.

The development of advanced systems concepts such as a hypersonic transatmospheric vehicle described will require many key elements to be in place before even a feasible design is possible. For an optimized design, the need to understand the proper theoretical context for the generalized second-law principle becomes critical. The proper context $[13,14]$ for implementing analysis and design principles based on the second law begins with the fundamental equation

$$
S(\bar{x})-S(x)-S^{\prime}(\bar{x}) \cdot(\bar{x}-x) \geq 0,
$$

where $S(x)$ represents the entropy functional and $x$ the dependent variable with reference state $\bar{x}$. A proper functional form for the entropy requires satisfaction of this inequality. The fundamental equation also provides a means for constructing the entropy balance equation in the infinitesimal time limit

$$
\lim _{\Delta t \rightarrow 0} \frac{S\left(x^{t+\Delta t}\right)-S\left(x^{t}\right)}{\Delta t}-S^{\prime}\left(x^{t+\Delta t}\right) \cdot\left(\frac{x^{t+\Delta t}-x^{t}}{\Delta t}\right) \geq 0,
$$

so that by replacing the governing equations for the time rate of change in the field variables, one identifies the terms representing entropy flux, entropy generation, and the time-rate of change in the entropy. We have previously shown that the fundamental concavity property for the entropy (1) becomes a convexity requirement on the exergy, providing two equally valid, complementary ways for stating and developing the second-law beyond its origins in thermodynamics.

The Euler (or Navier-Stokes) equations can be written in compact notation

$$
\frac{\partial \mathbf{q}}{\partial t}=-\frac{\partial \mathbf{f}_{j}}{\partial x_{j}}
$$

where $\mathbf{q}$ represents the state vector and $\mathbf{f}_{j}$ the algebraic flux vector of state quantities and we use the conventional omission of the summation sign with the repeated index notation. For an ideal gas the entropy formula is readily available from thermodynamics, such that $S=S(\mathbf{q})$ with respect to the state variables $\mathbf{q}=(\rho, \rho u, \rho v, \rho w, \rho e)$. The specific formula, from thermodynamics, is

$$
s(\rho, T)-s_{0}=c_{V} \ln \left(\frac{T}{T_{0}}\right)-R \ln \left(\frac{\rho}{\rho_{0}}\right) .
$$

The total specific energy is $\rho e=(1 / 2) \rho\left(u_{1}^{2}+u_{2}^{2}+u_{3}^{2}\right)+$ $p /(\gamma-1)$, so that the temperature can be written as

$$
R T(q)=(\gamma-1)\left\{\frac{q_{5}}{q_{1}}-\frac{1}{2}\left(\frac{q_{2}^{2}+q_{3}^{2}+q_{4}^{2}}{q_{1}^{2}}\right)\right\} .
$$

Setting the constant reference state $s_{0}$ equal to zero and writing $T$ in terms of the state variables give

$$
S(\mathbf{q})=\rho s=\frac{q_{1}}{\gamma-1} \ln \left\{\frac{\gamma-1}{2}\left[\frac{2 q_{1} q_{5}-\left(q_{2}^{2}+q_{3}^{2}+q_{4}^{2}\right)}{q_{1}^{\gamma+1}}\right]\right\} .
$$


The balance of entropy emerges from (2) and substituting the right-hand side of (3):

$$
\frac{\partial S}{\partial t}+\frac{\partial S}{\partial \mathbf{q}} \cdot \frac{\partial \mathbf{q}}{\partial t}=0 \longrightarrow \frac{\partial S}{\partial t}-\frac{\partial S}{\partial \mathbf{q}} \cdot \frac{\partial \mathbf{f}_{j}}{\partial x_{j}}=0 .
$$

If the fluxes contain only the convective transport of mass, momentum, and energy as represented in the Euler equations, then it is possible to show that

$$
\frac{\partial S}{\partial \mathbf{q}} \cdot \frac{\partial \mathbf{f}_{j}}{\partial x_{j}}=\frac{\partial F_{j}}{\partial x_{j}},
$$

where the entropy fluxes are $F_{j} \equiv \rho s u_{j}$ and the entropy generation rate

$$
\dot{S}_{\text {gen }}=\frac{\partial S}{\partial t}+\frac{\partial F_{j}}{\partial x_{j}}=0
$$

demonstrates that entropy generation is zero for adiabatic, inviscid (reversible) gas dynamics. If the fluxes in (3), however, contain the terms that model the effects of viscosity and heat conduction, then

$$
\frac{\partial S}{\partial \mathbf{q}} \cdot \frac{\partial \mathbf{f}_{j}}{\partial x_{j}}=\frac{\partial F_{j}}{\partial x_{j}}+\frac{\partial}{\partial x_{j}}\left(\frac{Q_{j}}{T}\right)-\frac{\tau_{j i}}{T} \frac{\partial u_{i}}{\partial x_{j}}+\frac{Q_{j}}{T^{2}} \frac{T}{\partial x_{j}},
$$

where $Q_{j}$ represents the heating rate per unit area (heat flux) and $\tau_{j i}$ the viscous stress tensor. On substitution in the entropy balance equation, we get two expressions that represent entropy generation:

$$
\dot{S}_{\mathrm{gen}}=\left\{\begin{array}{l}
\frac{\partial S}{\partial t}+\frac{\partial F_{j}}{\partial x_{j}}+\frac{\partial}{\partial x_{j}}\left(\frac{Q_{j}}{T}\right), \\
\frac{\tau_{j i}}{T} \frac{\partial u_{i}}{\partial x_{j}}-\frac{Q_{j}}{T^{2}} \frac{\partial T}{\partial x_{j}} .
\end{array}\right.
$$

The entropy generation rate then relates to the rate of exergy destruction via the so-called Guoy-Stodola theorem

$$
\dot{X}_{\mathrm{des}}=T_{0} \dot{S}_{\text {gen }}
$$

in honor of the two pioneers who advocated the utility of the concept. The quantity on the left-hand side of (13) represents the "lost work" resulting from irreversible mechanisms present in the simulated process. By coupling the numerical solution of the fluid flow equations with the added constraint that (13) must be satisfied according to the principle of nonnegative entropy generation, $\dot{S}_{\text {gen }} \geq 0$ one can gain insight into the details of the loss mechanisms of a particular process or design. In addition, this will greatly increase our understanding of the relative contribution of numerical error in computational simulations against the physical dissipation model of the governing equations.

Work in this direction couples the solution of the governing equations with the satisfaction of the second law of thermofluid dynamics as an additional constraint. A step towards this goal is to utilize the second law as an a posteriori check on the solution: to identify regions where exergy is destroyed or work-potential losses are excessive. For the Euler equations, entropy is strictly conserved but enforcing this constraint leads to nonunique possibly unnatural solutions. It has long been known that physically relevant solutions to the Euler equations require some kind of entropy fix, usually in the form of finite numerical dissipation. In fact, the more successful approaches contain leading-error terms that explicitly generate a finite amount of entropy proportional to velocity and temperature gradients, much like the physical model provided by viscosity and heat conduction in the Navier-Stokes equations. This allows solutions to the Euler equations to "capture" physically correct compressive shock waves. Conversely, when solving for Navier-Stokes equations, which contain entropy-producing mechanisms explicitly, too much entropy generation occurs when using numerical methods of low accuracy.

A numerical method applied to the solution of the governing equations aims at calculating the updated variables from the presently known distribution at a previous time step. The space and time integration of the balance equations can be separated so that the resulting finitevolume, semidiscrete formula

$$
\left(\frac{d q}{d t}\right)_{i}+\sum_{k=1}^{m} \frac{A_{k}}{V_{i}}(\hat{n} \cdot \vec{f})_{k}=0
$$

where $A_{k} / V_{i}$ represents the ratio of the cell-face area to the discrete cell volume, can be integrated in time to obtain new values of the state variables. For the Euler equations, the discrete entropy balance equation becomes

$$
\left(\dot{S}_{\text {gen }}\right)_{i}^{n+1-n}=\frac{S^{n+1}-S^{n}}{\Delta t}+\sum_{k=1}^{m} \frac{A_{k}}{V_{i}}(\hat{n} \cdot \vec{F})_{k}^{n+1}=0
$$

if the balance equations are integrated implicitly in time and

$$
\left(\dot{S}_{\text {gen }}\right)_{i}^{n \rightarrow n+1}=\frac{S^{n}-S^{n+1}}{\Delta t}+\sum_{k=1}^{m} \frac{A_{k}}{V_{i}}(\hat{n} \cdot \vec{F})_{k}^{n}=0
$$

if the balance equations are integrated explicitly in time (note the difference in the entropy terms and the exponent for the entropy fluxes). For the Navier-Stokes equations that model a viscous, heat-conducting fluid, some additional terms would appear, but the basic idea remains the same. A numerical method that satisfies the nonnegative entropy generation principle in discrete form can be said to satisfy the second law of thermodynamics. So by calculating and monitoring the magnitude and sign of the entropy generation locally, one may identify regions where the second law is violated (Figure 4). This allows an expanded capability for ensuring that the computational simulation satisfies physical theory, is numerically stable, and incurs a minimum of numerical error.

For the Euler equations, which represent the flow of an inviscid, adiabatic fluid, the entropy balance equation yields zero net entropy generation. However, under flow conditions where shocks are expected, entropy generation must be positive. This is why numerical "shock capturing" methods in CFD require a finite amount of numerical dissipation both for numerical stability and also to guarantee that only 
$S$ gen rate

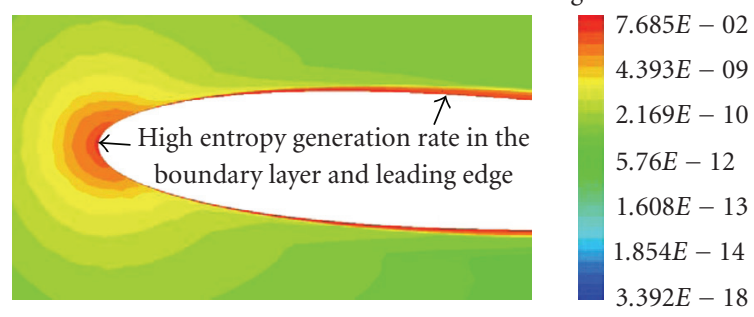

FIGURE 4: Entropy production rates computed with high-fidelity CFD code can be usefully identify regions of numerical error and where the second-law is violated. For system design, the computation would be a useful way of identifying regions of irreversible waste.

compressive shocks show up in the solution. For the NavierStokes equations, where viscosity and heat conduction are accounted for, the second law of thermodynamics yields two expressions for the entropy generation rate, one that serves as a constitutive constraint on the sign of the viscosity and heat conduction coefficient, and another which represents the transport of entropy with fluid flow, as shown in (12). It is possible to couple the entropy balance equation to the numerical solution of the Navier-Stokes equations by using the transport equation together with the constitutive relation that may be used as a constraint on the entropy generation rate itself. If enforced, the second law should thus provide a constraint on the entropy generation rate such that only physically possible solutions will be admitted. In addition, it may be possible to formulate the numerical solution so that the physical effects of fluid viscosity and heat conduction may be separated from numerical error. This represents a significant advance in CFD and provides the necessary guidelines to develop optimization procedures for aerospace vehicle design that minimize entropy generation rate and hence excess exergy destruction.

We seek to develop the computational capability to analyze systems in terms of the second law of thermodynamics. Since existing methods for modeling, simulating, analyzing, and designing aerospace vehicles rely solely on the conservation of energy, they fail to capture the subtle effects of the second law. More importantly, if we satisfy the first law of thermodynamics as a conservation of energy principle, we do not preclude the possibility of generating a design that violates the second law by creating a perpetual motion machine of the second kind. Including the second law of thermodynamics will then make it possible to perform numerical experiments for the synthesis and design of novel aerospace systems optimized according to a more complete physical theory. A generalized form of the second law beyond classical thermodynamics will thus provide the essential guidelines for developing the correct optimization principles for minimizing entropy-generation/exergy-destruction.

\section{The Future}

A very important factor in system development is the current emphasis on modeling and simulation-based acquisition.
There has to be an assumption that the models will be "sufficiently accurate". This subjective requirement means that the operational system is satisfactory. First, we can consider the possibilities of reduced emphasis on ground testing in favor of more emphasis on modeling and simulation in the development of any new system. Second is the desire to reduce the number of flight tests. The work in [15] not only discusses the rationale for these trends but also provides an indication of the recent state of the art in predicting aircraft characteristics. It contains seven pages of "Unanticipated Characteristics Discovered in Flight Test". The discussion covers experiences with a wide range of aircraft, commercial and military, fighters and transports, plus a wide range of characteristics. The anomalies also range from nuisance to significant, but there are many instances of failure to predict the aerodynamic characteristics, especially drag.

Modeling is a fundament of design; methods that enable the design and optimization of revolutionary aerospace vehicles lie outside existing, evolutionary approaches. Balancing performance and efficiency against cost and the capacity to sustain such vehicles will require a degree of control in energy conversion far beyond existing capabilities. Physical shape optimization may be possible with active flow control requiring the generation and manipulation of a plasma field or sheath. The fundamental physics of plasma flow are well known but its engineering implementation has yet to demonstrate its potential. For such a hypothetical vehicle, developing a unified analytical methodology falls to computer modeling and simulation requiring multiple disciplines. Such a unified approach may be based on the second law of thermodynamics, which provides both a universal concept (entropy) and a universal principle ideally suited to the task. The challenge and objective for this work is to show that such a new capability to perform multidisciplinary computational analyses is possible and advantageous.

\section{Conclusion}

The major purpose of this overall work has been to develop, understand, and apply entropy production as the criterion to evaluate vehicle performance and performance losses consistently. This forms the basis for the use of a "common currency" in the system-level vehicle design process-the use of energy concepts is one example of where entropic analysis may be of great use. Such capability may allow the development of new and innovative concepts that do not "just" marginally improve performance but may enable the realization of entire new regimes of performance and operability, especially for high-speed aerospace vehicles. The overall vision for this research is to prove that a complete integration of exergy-based methods promises to facilitate a breakthrough in optimization of aerospace vehicles based on a system of energy systems. The current evolutionary designs have achieved a high degree of efficiency building on previous experiences. Evolutionary methods only work, however, when the configuration under consideration is close to the existing experience and database. We have proposed a 
foundation for system-level design with optimization based on minimum exergy destruction. The potential of these new methods will be realized when we attempt the next revolution for which there is no previous flight experience.

\section{Acknowledgments}

The authors gratefully acknowledge the support of the US Air Force Office of Scientific Research under the program management of Dr. Victor Giurgiutiu (Structural Mechanics) and the USAF Small Business Innovative Research (SBIR) Program for funding to our industrial partners.

\section{References}

[1] D. J. Moorhouse, "The multidisciplinary engineer in the context of concurrent engineering," in Proceedings of the Aerodynamic Design and Optimization of Flight Vehicles in a Concurrent Multi-Disciplinary Environment, RTO Meeting Proceedings, June 2000, RTO-MP-35.

[2] D. J. Moorhouse, "A proposed system-level multidisciplinary analysis technique based on exergy methods," Journal of Aircraft, vol. 40, no. 1, pp. 11-15, 2003.

[3] J. R. Munoz and M. R. von Spakovsky, "A decomposition approach for the large scale synthesis/design optimization of highly coupled, highly dynamic energy systems," International Journal of Applied Thermodynamics, vol. 4, no. 1, pp. 19-33, 2001.

[4] D. F. Rancruel and M. R. von Spakovsky, "Use of a unique decomposition strategy for the optimal synthesis/design and operation of an advanced fighter aircraft system," in Proceedings of the 10th AIAA/ISSMO Multi-Disciplinary Analysis and Optimization Conference, Albany, NY, USA, August-September 2004.

[5] D. F. Rancruel and M. R. von Spakovsky, "Decomposition with thermoeconomic isolation applied to the optimal synthesis/design and operation of an advanced tactical aircraft system," Energy, vol. 31, no. 15, pp. 3327-3341, 2006.

[6] D. F. Rancruel and M. R. von Spakovsky, "Decomposition with thermoeconomic isolation applied to the optimal synthesis/design of an advanced tactical aircraft system," International Journal of Thermodynamics, vol. 6, no. 3, pp. 93105, 2003.

[7] D. F. Rancruel and M. R. von Spakovsky, "Use of a unique decomposition strategy for the optimal synthesis/design and operation of an advanced fighter aircraft system," in Proceedings of the 10th AIAA/ISSMO Multi-Disciplinary Analysis and Optimization Conference, Albany, NY, USA, August-September 2004.

[8] D. F. Rancruel and M. R. von Spakovsky, "A decomposition strategy applied to the optimal synthesis/design and operation of an advanced fighter aircraft system: a comparison with and without airframe degrees of freedom," in Proceedings of the ASME International Mechanical Engineering Congress (IMECE '03), vol. 43, pp. 477-490, Washington, DC, USA, November 2003, ASME paper no. 44402.

[9] V. Periannan, M. R. von Spakovsky, and D. J. Moorhouse, "Investigation of the effects of various energy and exergybased figures of merit on the optimal design of a high performance aircraft system," in Proceedings of the International Mechanical Engineering Congress and Exposition (IMECE '06),
Chicago, Ill, USA, November 2006, ASME paper no. IMECE2006-14186.

[10] D. Riggins, T. Taylor, L. Terhune, and D. Moorhouse, "Methods for the design of energy efficient high speed aerospace vehicles," The Aeronautical Journal, vol. 111, no. 1119, pp. 297309, 2007.

[11] K. Alabi, et al., "Multi-level exergy modeling, analysis and optimization for the integrated synthesis/design of aerospace systems," in Proceedings of the 25th Congress of the International Council of the Aeronautical Sciences (ICAS '06), Hamburg, Germany, September, 2006, ICAS paper 1.9.2.

[12] K. Alabi, F. Ladeinde, M. von Spakovsky, D. Moorhouse, and J. Camberos, "Assessing CFD modeling of entropy generation for the air frame subsystem in an integrated aircraft design/synthesis procedure," in Proceedings of the 44th AIAA Aerospace Sciences Meeting and Exhibit, Reno, Nev, USA, January 2006, AIAA paper 2006-0587.

[13] J. A. Camberos, "On the construction of entropy balance equations for arbitrary thermophysical processes," in Proceedings of the 39th AIAA Aerospace Sciences Meeting, January 2001, AIAA paper no. 2001-0815.

[14] J. A. Camberos, "Entropy concavity, the second-law, and the concept of exergy in thermophysics," in Proceedings of the 33rd AIAA Thermophysics Conference, Norfolk, Va, USA, June 2000, AIAA no. paper 99-3557.

[15] W. J. Norton, "Balancing modelling and simulation with flight test in military aircraft development," in Proceedings of the Advances in Flight Testing, December 1997, AGARD-CP-593. 

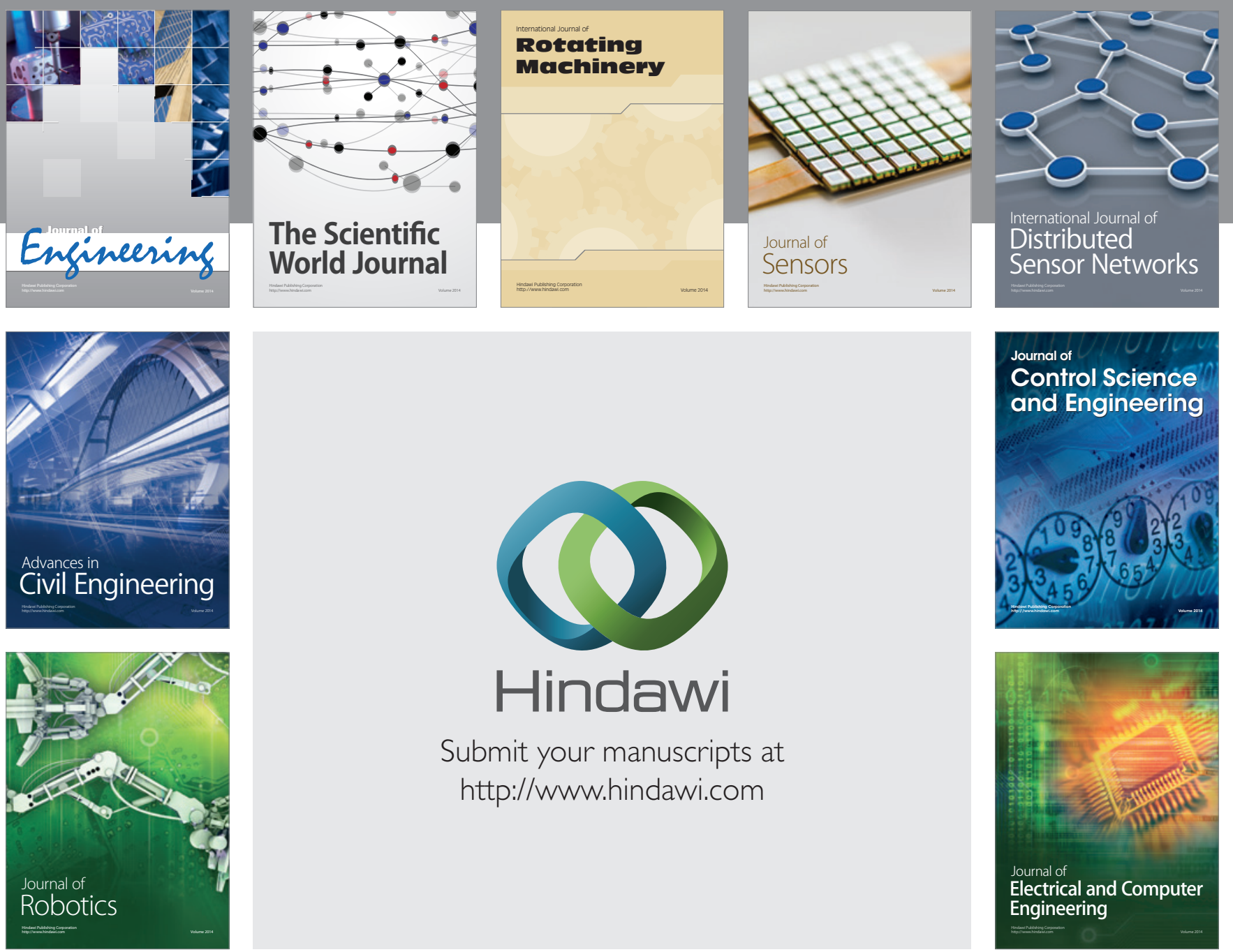

Submit your manuscripts at

http://www.hindawi.com
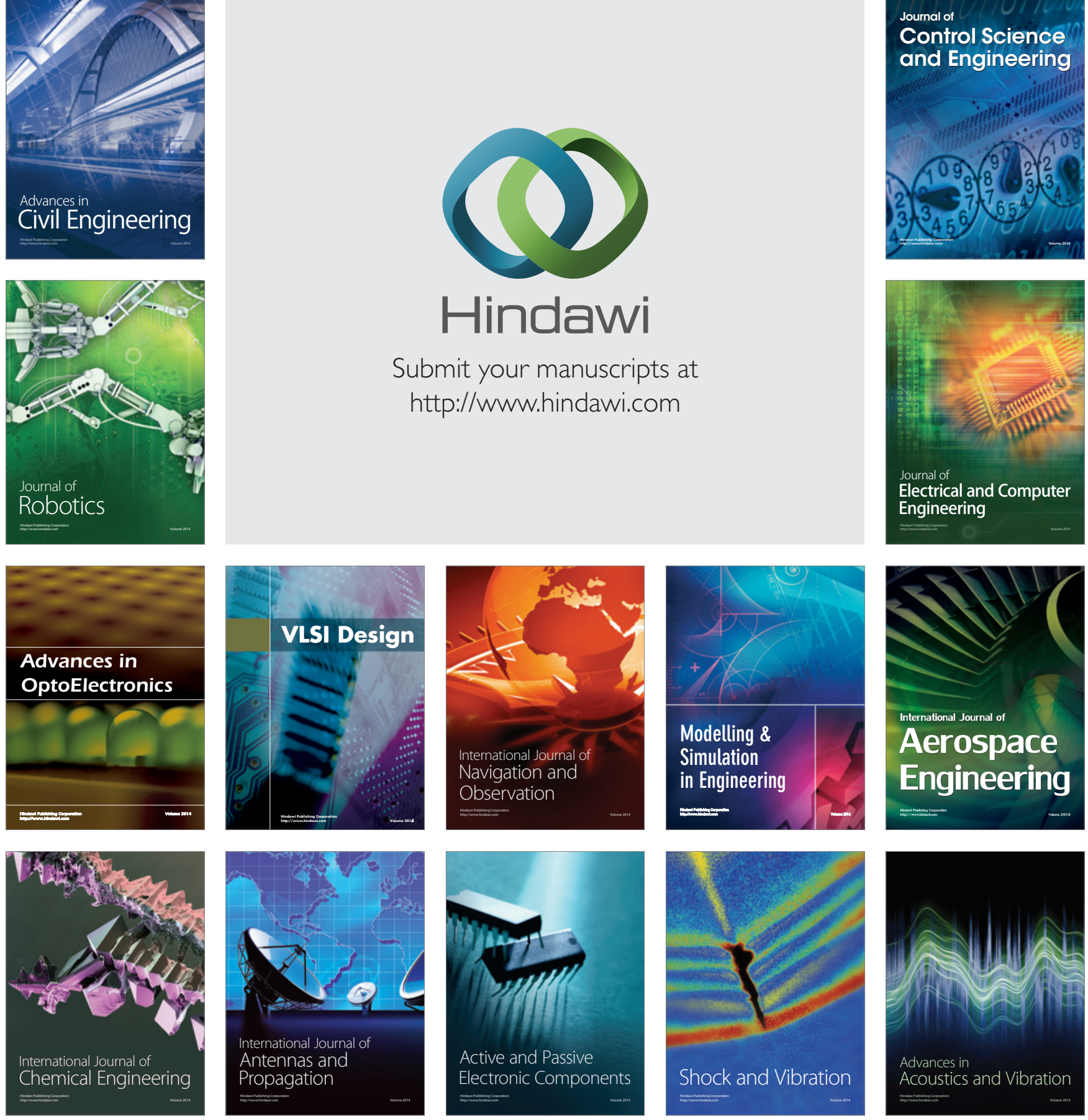